\title{
COMMENTARY
}

\section{Rapid diagnosis of sepsis using biomarker signatures}

\author{
Nigel James Silman \\ See related research by Ware et al., http://ccforum.com/content/17/5/R253
}

\begin{abstract}
Diagnosis of sepsis is complicated by non-specific clinical definitions and delays in laboratory analysis using tests which may have very poor predictive values. The use of host biomarker signature sets, which when measured in combination have high predictive values, offers a paradigm shift forwards for rapid, near-patient diagnosis. These analyses more closely mirror the rapid blood chemistry and hematology analyses which often are used for near-patient testing and diagnosis.
\end{abstract}

Diagnosis of severe sepsis is one area in which a laboratory confirmation could positively affect patient management but, owing to a lack of clear correlation between single biomarkers and disease, is often of little value. Sepsis is a significant health issue in many countries where invasive surgical procedures, for example, have become more frequent. Over 170 different biomarkers have been described as either diagnostic or prognostic for sepsis, but clinical trials in which these biomarkers are evaluated singly often give relatively low positive predictive value outcomes and tests based upon single biomarkers are therefore of limited value since diagnosis is made using clinical observations, and prophylactic antimicrobial therapy often is used as a preventative measure in case of infectious etiology.

\section{Biomarker signatures}

Recent discovery work on diagnostic biomarkers for sepsis concentrates efforts on two different approaches; the first is on using multiple host biomarker changes to define a 'signature' which is indicative of disease. In the previous issue of Critical Care, Ware and colleagues

Correspondence: nigel.silman@phe.gov.uk

Public Health England, Manor Farm Road, Porton Down, Salisbury, Wiltshire SP4 0JG, UK describe a clinical study using multiple host biomarkers to aid diagnosis of acute respiratory distress syndrome (ARDS), a particular complication of sepsis which is often under-diagnosed because of the difficulties in applying a non-specific definition of disease [1]. All of the patients enrolled in this clinical study had a diagnosis of severe sepsis, and $50 \%$ also met the definition of ARDS.

Host biomarker signatures with potential for use as laboratory diagnostic markers can be either proteins which are easily measured, as in this case, or nucleic acids. The latter approach was recently described by $\mathrm{Ma}$ and colleagues [2], and microRNAs were sequenced and used to provide signature sequences which were shown to clearly distinguish between sepsis and systemic inflammatory response syndrome. The alternative to measuring changes in host biomarker(s) is the direct detection of the pathogen; one of the key microbiological analyses recommended for diagnosis of severe sepsis is the collection of blood cultures prior to commencement of broad-spectrum antimicrobial therapy and isolation of the pathogen [3] or detection of the pathogen nucleic acids by polymerase chain reaction assay. The direct detection of pathogens or nucleic acids in samples often provides little guidance to the clinician because of either delays while samples are transported to and analyzed in the laboratory or the sometimes low positive predictive value of such analyses which, therefore, cannot be used definitively as 'rule out' diagnostics.

Although a number of sepsis diagnostic kits based on single biomarkers are commercially available, the value of the results obtained is limited and often does not clearly inform patient management. Here is the opportunity for biomarker signature sets. The advent of rapid diagnostic platforms means that, to start with, multiple biomarkers can be analyzed in parallel in a reproducible and rapid manner; such technologies have been available for blood chemistry and hematology for many years now, and it is high time microbiology caught up. Measurement of host biomarkers more closely resembles the 
analyses which are performed by clinical chemistry and hematology labs and which frequently are performed in intensive treatment units so that patient management can be rapidly changed on the basis of the results obtained. The major problem with measurement of host biomarkers and their ultimate use as diagnostic or prognostic indicators of infectious disease is not one of technologies, as these are plentiful, but of data interpretation. The difficulty arises not from the absolute measurement of such serum biomarkers, as described by Ware and colleagues [1], as this is very straightforward, but in analysis and assignment of a positive or negative test result, which is where the work now needs to be done. As we know, single biomarkers are often of low predictive value in the diagnosis of complicated conditions such as sepsis, not because they are difficult parameters to measure, but because it is not always clear when a biomarker is elevated (and when it is not) when used in the outbred population that humans are. The same issue of data interpretation occurs with analysis of multiple biomarkers, as an elevated level of a single biomarker can be abnormal in one person but normal in another. However, this is where the signature approach comes in; a large number of both 'normal' (that is, healthy) patient samples as well as samples from those diagnosed and proven to have the condition under investigation (in this case, sepsis) need to be analyzed and these data then need to be used to set the cutoff values for each biomarker and for the signature set so that high positive predictive and negative predicative values can be accurately assigned. The ability to rapidly measure multiple biomarkers, coupled with well-validated data sets, not only means that diagnosis of sepsis can for the first time be moved nearer to the patient but also has high value in subsequent patient management and therefore prognosis.

\section{Conclusions}

Ware and colleagues describe the measurement of a number of host biomarkers, and selection of the five with the highest diagnostic and prognostic value, to be used in combination as a signature set. Such host biomarker signatures offer considerable advantages in that they can be analyzed, not in remote laboratories, but using rapid, near-patient technologies to positively improve patient management and diagnosis of complex diseases such as sepsis.

Abbreviation

ARDS: Acute respiratory distress syndrome.

Competing interests

The author declares that he has no competing interests.
Published: 04 Dec 2013

\section{References}

1. Ware LB, Koyama T, Zhao Z, Janz DR, Wickersham N, Bernard GR, May AK, Calfee CS, Matthay MA: Biomarkers of lung epithelial injury and inflammation distinguish severe sepsis patients with acute respiratory distress syndrome. Crit Care 2013, 17:R253.

2. Ma Y, Vilanova D, Atalar K, Delfour O, Edgeworth J, Ostermann M, Hernandez-Fuentes M, Razafimahatratra S, Michot B, Persing DH, Ziegler I, Törös B, Mölling P, Olcén P, Beale R, Lord GM: Genome-wide sequencing of cellular microRNAs identifies a combinatorial expression signature diagnostic of sepsis. PLoS One 2013, 8:e75918.

3. Schmitz RP, Keller PM, Baier M, Hagel S, Pletz MW, Brunkhorst FM: Quality of blood culture testing - a survey in intensive care units and microbiological laboratories across four European countries. Crit Care 2013, 17:R248.

$10.1186 / \mathrm{cc} 13137$

Cite this article as: Silman: Rapid diagnosis of sepsis using biomarker signatures. Critical Care 2013, 17:1020 